\title{
Prevention of colon cancer by pre- and probiotics: evidence from laboratory studies
}

\author{
Bandaru S. Reddy \\ American Health Foundation, Valhalla, New York 10595, USA
}

\begin{abstract}
Oligofructose and inulin, selective fermentable chicory fructans, have been shown to stimulate the growth of bifidobacteria which are regarded as beneficial strains in the colon. Studies were designed to evaluate inulin (Raftiline) and oligofructose (Raftilose), for their potential inhibitory properties against aberrant crypt foci (ACF) formation in the colon of rats. ACF are putative preneoplastic lesions from which adenomas and carcinomas may develop. The results of this study demonstrate that dietary administration of oligofructose and inulin inhibits the formation of preneoplastic lesions in the colon suggesting the potential colon tumour inhibitory properties of chicory fructans. Since these prebiotics selectively stimulate the growth of bifidobacteria, tumour inhibitory activity of lyophilized cultures of Bifidobacterium longum (BL) against azoxymethane (AOM)-induced colon carcinogenesis in rats and modulating effect of these cultures on colonic tumour cell proliferation, ornithine decarboxylase (ODC) activity, and ras-p21 oncoprotein expression were investigated. Dietary administration of lyophilized cultures of BL strongly suppressed AOM-induced colon tumour development. Inhibition of colon carcinogenesis was associated with a decrease in colonic mucosal cell proliferation and colonic mucosal and tumour ODC and ras-p21 activities.
\end{abstract}

Colon cancer: Oligofructose: Inulin: Bifidobacterium longum

Colorectal cancer is one of the leading causes of cancer mortality in both men and women in the Western countries including the USA (Parker et al. 1997). Epidemiological studies indicate that increased consumption of fruits and vegetables and high total dietary fibre reduce the risk of development of colon cancer (Howe et al. 1992). Human metabolic studies suggest that beneficial effects of dietary fibre in relation to colon cancer development depends on the composition and physical properties of fibre (Reddy et al. 1992). Animal model studies also demonstrate that tumourinhibitory properties of dietary fibre in the colon depends on its composition (Reddy, 1995). Among the types of dietary fibre, inulin and oligofructose are $\beta-(2-1) D$ fructans which are fermented by colonic microflora and behave as soluble fibres (Gibson \& Roberfroid, 1995). Oligofructose and inulin which occur in common foodstuffs such as chicory, leeks, garlic, onion, artichoke and asparagus at high levels selectively stimulate the growth of bifidobacteria at the expense of bacteroides, clostridia or coliforms which are maintained at low levels (Gibson \& Roberfroid, 1995; Gibson et al. 1995). Bacterial fermentation of chicory fructans and other oligofructoses produces short-chain fatty acids (SCFA) in the colon including a small amount of butyric acid (Gibson \& Roberfroid, 1995; Campbell et al. 1997) which has been shown to increase apoptosis in the colon (Hague et al. 1993). Furthermore, there are studies to demonstrate that cultures of bifidobacteria increase the host's immune response (Sekine et al. 1995). These observations raise the possibility that selective fermentable non-digestible oligosaccharides that enhance the growth of bifidobacteria in the gut could potentially inhibit colon carcinogenesis. It was therefore of interest to evaluate the inhibitory properties of dietary oligofructose, inulin and bifidobacteria against colon carcinogenesis.

\section{Oligofructose and inulin}

This study was designed to determine the potential inhibitory properties of oligofructose (Raftilose) and inulin (Raftiline) on azoxymethane (AOM)-induced colon carcinogenesis in male F344 rats using colonic aberrant crypt foci (ACF) as the endpoint (Reddy et al. 1997). ACF, which are recognized as early preneoplastic lesions in the colon, have consistently been observed in experimentally induced colon carcinogenesis in laboratory animals and in the colonic mucosa of patients with colon cancer (McLellan et al. 1991; Pretlow et al. 1992). Aberrant crypts are putative precursor lesions from which adenomas and carcinomas may develop in the colon. ACF express mutations in the apc gene and ras oncogene that appear to be involved in colon cancer development (Vivona et al. 1993). Several inhibitors of ACF formation have been shown to reduce the incidence of colon tumours in laboratory animals (Wargovich et al. 1996) suggesting that ACF induction can be used to evaluate novel agents for their potential chemopreventive properties against colon cancer.

In this study, groups of 7-week-old male F344 rats were fed the AIN-76A (control) and the experimental diets 
Table 1. Effect of dietary oligofructose and inulin on colonic ACF formation in male F344 rats*

\begin{tabular}{|c|c|c|c|c|c|}
\hline \multirow[b]{2}{*}{ Experimental group } & \multirow[b]{2}{*}{ Total ACF/colon } & \multicolumn{3}{|c|}{ Foci containing number of aberrant crypts } & \multirow[b]{2}{*}{$\begin{array}{c}4 \text { or more } \\
\text { crypts/focus }\end{array}$} \\
\hline & & $1 \mathrm{crypt} /$ focus & 2 crypts/focus & 3 crypts/focus & \\
\hline Control diet & $120 \pm 28 \dagger$ & $19 \cdot 5 \pm 7 \cdot 3$ & $43 \cdot 7 \pm 7 \cdot 8$ & $28 \cdot 2 \pm 7 \cdot 5$ & $28 \cdot 3 \pm 8 \cdot 2$ \\
\hline Oligofructose, $10 \%$ & $\begin{array}{c}92 \pm 28 \ddagger \\
(P<0.024)\end{array}$ & $15 \cdot 4 \pm 7 \cdot 5$ & $\begin{array}{c}31.2 \pm 13 \ddagger \\
(P<0.01)\end{array}$ & $\begin{array}{c}21.3 \pm 7.8 \ddagger \\
(P<0.04)\end{array}$ & $23 \cdot 9 \pm 8 \cdot 2$ \\
\hline Inulin, $10 \%$ & $\begin{array}{c}78 \pm 37 \ddagger \\
(P<0.006)\end{array}$ & $15 \cdot 7 \pm 8 \cdot 2$ & $\begin{array}{c}24 \pm 12 \ddagger \\
(P<0.0001)\end{array}$ & $\begin{array}{c}16.6 \pm 7.2 \ddagger \\
(P<0.02)\end{array}$ & $21 \cdot 8 \pm 14.2$ \\
\hline
\end{tabular}

-Reddy et al. (1997)

tMean \pm SD.

‡Significantly different from the control diet. The level of significance is shown in parentheses.

containing $10 \%$ oligofructose (Raftilose) or inulin (Raftiline). At 7 weeks of age, all animals received s.c. injection of AOM dissolved in normal saline at a dose rate of $15 \mathrm{mg} /$ $\mathrm{kg}$ body weight, once weekly for 2 weeks. The animals were necropsied 7 weeks after the last AOM injection, and the ACF were visualized under light microscopy in the formalin-fixed, unsectioned methylene blue-stained colons. They were distinguished by their increased size, more prominent epithelial cells and pericryptal space. AOM treatment induced on the average $\sim 120 \mathrm{ACF} /$ colon (Table 1) ACF were predominantly observed in the distal colons. Efficacy endpoints used in this study were inhibition of the total number of $\mathrm{ACF} / \mathrm{colon}$ as well as the reduction of the number of multicrypt clusters $(\geq 2)$ of aberrant crypts/focus. Administration of oligofuctose or inulin in the diet significantly inhibited the total number of ACF/colon as compared to the control diet; the degree of inhibition was more pronounced in the animals fed inulin $(P<0.0006)$ than in those fed oligofructose $(P<0 \cdot 02)$. Crypt multiplicity in terms of two or three aberrant crypts/focus were also significantly inhibited in animals fed inulin $(P<0.02-$ $0.0001)$ and oligofructose $(P<0.04-0.01)$. These findings suggest that chicory fructan supplements inhibit ACF formation, an early preneoplastic marker of malignant potential in the process of colon carcinogenesis. The mechanisms by which oligofructose and inulin inhibit preneoplastic lesions of the colon may involve the modulation of microflora (Gibson \& Roberfroid, 1995; Gibson et al. 1995) in the colon. In vitro studies showed that incubation of faecal bacterial cultures with oligofructose and inulin selectively stimulated the growth of bifidobacteria while maintaining the Escherichia coli or clostridia at low levels (Wang \& Gibson, 1993). Gibson et al. (1995) demonstrated that dietary administration of oligofructose or inulin significantly increased faecal bifidobacteria, whereas Bacteroides, clostridia and fusobacteria and/or Gram-positive cocci were decreased on total faecal bacterial count. The colonizing cells of bifidobacteria produce lactic acid, thereby lowering the intestinal $\mathrm{pH}$ to create a bacteriocidal environment for putative enteropathogens such as $E$. coli and $C$. perfringens thus developing a favourable microenvironment in the gut. This favourable microenvironment may also involve the modulation of bacterial enzymes such as $\beta$-glucuronidase that can convert procarcinogens to proximate carcinogens (Kulkarni \& Reddy, 1994). In addition to selective modulation of bifidobacteria, oligofructose and inulin increase the production of SCFA especially butyrate in the colon by microbial fermentation (Gibson \& Roberfroid, 1995). Although the production of butyrate is around $5 \%$ of total SCFA, it is of particular interest because it inhibits proliferation of a number of cell types in vitro and induces a more differentiated phenotype including colorectal tumor cells (Gamet et al. 1992).

\section{Bifidobacterial cultures}

Recent study from our laboratory demonstrates that dietary intake of lyophilized cultures of Bifidobacterium longum (BL) significantly suppressed the development of AOMinduced ACF in the colon (Kulkarni \& Reddy, 1994). It was therefore of interest to evaluate the colon tumour-inhibitory properties of dietary BL in the colon cancer model. The effects of dietary BL on AOM-induced as well as 2-amino-3methylimidazo[4,5- $f$ ]quinoline (IQ)-induced colon tumourgenesis were analysed in male F344 rats (Reddy \& Rivenson, 1993; Singh et al. 1997). We have also examined how this enterobacterial culture influences ornithine decarboxylase (ODC) activity, cell proliferation and the expression of mutated as well as normal cellular ras-p21 during AOMinduced colon carcinogenesis, in order to better understand the underlying mechanisms (Singh et al. 1997).

\section{Effect on AOM-induced colon carcinogenesis}

Male F344 rats were fed the modified AIN-76A diet containing 0 or $2 \%$ lyophilized cultures of $\mathrm{BL}$ and administered s.c. AOM dissolved in normal saline at a dose rate of $15 \mathrm{mg} /$ $\mathrm{kg}$ body weight, once weekly, for 2 weeks. Vehicle controls received s.c. equal volume of normal saline. Animals were maintained on control or experimental diets until termination of the study. Animals intended for analysis of cell proliferation were sacrificed 20 weeks after the second AOM injection, whereas animals intended for colon tumour histopathology and measurement of ODC activity and ras-p21 expression were sacrificed 40 weeks after last AOM injection. Mucosal cell proliferation, ODC activity and differential expression of total as well as mutant ras-21 were measured as described (Singh et al. 1997).

Table 2 summarizes the AOM-induced tumours in the colon in terms of tumour incidence (percentage of animals with tumours) and colon tumour multiplicity (number of tumours/animal). Dietary administration of $\mathrm{BL}$ cultures 
Table 2. Inhibitory effect of lyophilized cultures of Bifidobacterium longum on AOM-induced colon carcinogenesis in F344 rats*

\begin{tabular}{|c|c|c|c|c|c|c|c|}
\hline \multirow[b]{2}{*}{$\begin{array}{l}\text { Dietary } \\
\text { regimen }\end{array}$} & \multicolumn{2}{|c|}{ Colon tumourigenesis } & \multicolumn{3}{|c|}{ Colon mucosa } & \multicolumn{2}{|c|}{ Colon tumors } \\
\hline & $\begin{array}{c}\% \text { animals } \\
\text { with tumours }\end{array}$ & Tumours/animal & $\begin{array}{l}\text { Labelling index } \\
(\%)\end{array}$ & ODC activity & $\begin{array}{l}\text { Total ras-p21 } \\
\text { activity }\end{array}$ & ODC activity & $\begin{array}{l}\text { Total ras-p21 } \\
\text { activity }\end{array}$ \\
\hline $\begin{array}{l}\text { Control diet } \\
2 \% \mathrm{BL}\end{array}$ & $\begin{array}{c}77 \\
53 \ddagger\end{array}$ & $\begin{array}{r}1.8 \pm 1.27 \dagger \\
0.83 \pm 0.98 \ddagger\end{array}$ & $\begin{array}{l}18 \cdot 9 \pm 1 \cdot 1 \\
12 \cdot 8 \pm 1 \cdot 1 \neq\end{array}$ & $\begin{array}{l}66 \pm 10 \\
32 \pm 6 \ddagger\end{array}$ & $\begin{array}{c}14.2 \pm 3.0 \\
8.9 \pm 2.7 \S\end{array}$ & $\begin{array}{l}456 \pm 147 \\
101 \pm 309\end{array}$ & $\begin{array}{l}32 \cdot 7 \pm 5 \cdot 9 \\
19 \cdot 8 \pm 4 \cdot 8 \S\end{array}$ \\
\hline
\end{tabular}

'Singh et al. (1997).

†Mean \pm SD.

tSignificantly different from control diet. $P<0.05$.

$\S$ Significantly different from control diet, $P<0.01$.

iSignificantly different from control diet, $P<0.001$.

significantly inhibited the incidence of colon adenocarcinomas $(P<0.05)$, and colon tumour multiplicity in terms of tumours/animal $(P<0.001)$.

In addition, colon tumour inhibitory property of lyophilized cultures of BL was associated with the inhibition of colonic mucosal cell proliferation, and suppression of ODC activity and expression of total and mutated ras-p21, in the colonic mucosa and tumours as compared to that in control diet (Table 2). Biasco et al. (1991) observed a significant decrease in mucosal cell proliferation in upper colonic crypts of patients with colon adenomas after the administration of $L$. acidophilus and $B$. bifidus cultures. Elevated levels of ODC activity have been reported in neoplastic human colon versus normal appearing colonic mucosa (Porter et al. 1987; Singh et al. 1992), in dysplastic polyps versus non-dysplastic polyps (Luk \& Baylin, 1984) and also in non-involved mucosa from polyposis patients $v$. non-involved mucosa from normal individuals (Luk et al. 1989). Similarly, ODC activity has been found to be consistently higher in non-familial colon adenocarcinomas compared to adjacent mucosa. Evidence that enhanced ODC activity may play an important role in colon tumourdevelopment is provided by the observation that difluoromethylornithine, a high specific and irreversible inhibitor of ODC, suppressed colon tumour development in a time-dependent manner in carcinogen-treated rodent (Singh et al. 1992). In the current study, we observed elevated levels of ODC activity both in colon tumours and

Table 3. Inhibitory effects of lyophilized cultures of Bifidobacterium longum on 2-amino-3-methylimidazo[4,5- $f$ ]quinoline-induced tumourigenesis in F344 rats*

\begin{tabular}{|c|c|c|c|c|}
\hline \multirow[b]{2}{*}{ Dietary regimen } & \multicolumn{2}{|c|}{ Colon } & \multicolumn{2}{|c|}{ Mammary gland } \\
\hline & Incidence & Multiplicity $\ddagger$ & Incidence & † Multiplicity \\
\hline $\begin{array}{l}\text { Male rats } \\
\text { Control diet } \\
0.5 \% \mathrm{BL}\end{array}$ & $\begin{array}{l}27 \\
09\end{array}$ & $\begin{array}{c}0.43 \pm 0.89 d \\
0.9\end{array}$ & $\begin{array}{l}0 \\
0\end{array}$ & $\begin{array}{l}0 \\
0\end{array}$ \\
\hline $\begin{array}{l}\text { Female rats } \\
\text { Control diet } \\
0.5 \% \mathrm{BL}\end{array}$ & $\begin{array}{l}0 \\
0\end{array}$ & $\begin{array}{l}0 \\
0\end{array}$ & $\begin{array}{l}27 \\
13\end{array}$ & $\begin{array}{c}0.46 \pm 0.80 \\
0.19 \pm 0.469\end{array}$ \\
\hline
\end{tabular}

"Reddy and Rivenson (1993).

†Percentage of animals with tumours.

$\ddagger$ Tumours/animal.

$\S$ Mean \pm SD

ISignificantly different from its respective control diet in the same gender, $P<0.05$. in uninvolved colonic mucosa of AOM-treated animals (Singh et al. 1997). In addition, ODC activity was significantly decreased in colonic mucosa as well as in colon tumours of AOM-treated animals administered lyophilized cultures of BL. Although, the precise mechanism of inhibition of ODC activity by dietary BL cultures is not clear, it is likely that these effects may proceed through diverse physiological and metabolic alterations.

Ras activation represents one of the earliest and most frequently occurring genetic alterations associated with human cancers, especially cancer of the colon (Barbacid, 1990). Elevated levels of ras-p21 have been correlated with increased cell proliferation, histological grade, nuclear anaplasia and degree of undifferentiation (Kotsinas et al. 1993). In experiments where mutated ras genes are selectively inactivated, the pre-existing tumour phenotype reverts to a more normal form, indicating that activated ras may be necessary for the maintenance of malignant behaviour (Mukhopadhya et al. 1991). As regards the mechanism of inhibition of ras activation afforded by BL cultures, it is hypothesized that bifidobacterial cells, as a biological response modifier, modulate the induction of the methylguanine repair protein, $\mathrm{O}^{6}$-methylguanine DNA methyltransferase, which acts as a suicide enzyme that stochiometrically accepts a methyl group onto itself, restoring the original guanine in DNA by in situ demethylation (Pegg \& Dolan, 1989). To our knowledge, there are no other data pertaining to the modulation of ras function by lactic cultures. It is, however, clear from our results that BLaugmented suppression of AOM-induced ras activity may interfere with the progression of events leading to colon tumour development.

\section{Effect of Bifidobacterium longum on 2-amino-3- methylimidazo $[4,5-f]$ quinolin-induced colon carcinogenesis}

The formation of mutagens upon broiling fish and meat was first discovered by Sugimura et al. (1977). IQ, a heterocyclic aromatic amine produced from food pyrolysis, was first isolated from broiled fish. Subsequently, it was isolated from a variety of broiled or cooked fish and meat (Kasai $e t$ al. 1980). IQ has a multitarget organospecificity with specific cancer induction in Zymbal gland, skin, colon. oral cavity, and mammary gland of rodents (Sugimura et al. 1991). Although it is not clear whether these heterocyclic amines may contribute to human cancer 
development, it is certain that these compounds are present in cooked foods and pose a credible risk to humans.

Because IQ induces colon tumours in male and female rats, and bacterial cultures that ferment milk possess anticarcinogenic properties, the possibility exists that these bacterial cultures may prevent IQ-induced carcinogenesis. Accordingly the inhibitory effect of lyophilized cultures of $\mathrm{BL}$ on IQ-induced carcinogenesis was investigated in male and female F344 rats (Reddy \& Rivenson, 1993). Beginning at 5 weeks of age, male and female rats were divided into various experimental groups and fed one of the high-fat, semipurified diets containing 0 and $0.5 \%$ lyophilized cultures of BL with or without 125 p.p.m. IQ in the diet. All animals were continued on this regimen until the termination of the study at week 58 .

The results indicated that lyophilized cultures of $\mathrm{BL}$ significantly inhibited the IQ-induced incidence (percentage of animals with tumours) of colon ( $100 \%$ inhibition) tumours and multiplicity (tumours/animal) of colon in male rats (Table 3 ). In female rats, dietary supplement of BL cultures also suppressed the IQ-induced mammary carcinogenesis to $50 \%$ of those observed in animals fed the control diet. The mammary tumour multiplicity (tumours/animal) was significantly $(P<0.05)$ inhibited in female rats fed the diet containing Bifidobacterium cultures. These findings suggest that Bifidobacterium supplements in the diet inhibit IQ-induced colon tumours and to a lesser extent mammary tumours in F344 rats.

In summary, the results thus far generated demonstrate that dietary administration of prebiotics such as oligofructose and inulin and lyophilized cultures of BL inhibits the formation of preneoplastic lesions in the colon and colon and/or mammary carcinogenesis in laboratory animal models.

\section{References}

Barbacid M (1990) Ras oncogenes: their role in neoplasia. European Journal of Clinical Investigation, 20, 225-235.

Biasco G, Paganelli GM \& Brandi G (1991) Effect of Lactobacillus acidophilus and Bifidobacterium bifidum on rectal cell kinetics and fecal pH. Italian Journal of Gastroenterology 23, 142.

Campbell JM, Fahey Jr GC \& Wolf BW (1997) Selected indigestible oligosaccharides affect large bowel mass, cecal and fecal short-chain fatty acids, $\mathrm{pH}$ and micorflora in rats. Journal of Nutrition 127, 130-136.

Gamet L, DaVaind D, Denis-Poouxviel C, Ramsey C \& Murat JC (1992) Effects of short-chain fatty acids on growth and differentiation of the human colon cancer cell line HT 29. International Journal of Cancer 52, 286-289.

Gibson GR \& Roberfroid MB (1995) Dietary modulation of the human colon microbiota: introducing the concept of prebiotics. Journal of Nutrition 125, 1401-1412.

Gibson GR, Beatty ER, Wang X \& Cummings JH (1995) Selective stimulation of bifidobacteria in the human colon by oligofructose and inulin. Gastroenterology 108, 975-982.

Hague A, Manning AM, Hanlon KA, Hutschtscha LI, Hart D \& Paraskeva C (1993) Sodium butyrate induces apoptosis in human colonic tumor cell lines in p53-independent pathway: implications for possible role of dietary fiber in the prevention of large bowel cancer. International Journal of Cancer 55, 498505 .

Howe GR, Benito E, Castello R et al. (1992) Dietary intake of fiber and decreased risk of cancers of the colon and rectum: evidence from the combined analysis of 13 case-control studies. Journal of the National Cancer Institute 84, 1887-1896.

Kasai H, Nishimura S, Wakabayashi K, Nagao M, \& Sugimura T (1980) Chemical synthesis of 2-amino-3-methylimidazo [4,5-f]quinoline (IQ), a potent mutagen isolated from broiled fish. Proceedings of the Japanese Academy 56B, $382-384$.

Kotsinas A, Spandidos DA, Romanowski P \& Wyllie AH (1993) Relative expression of wild-type and activated $\mathrm{Ki}$-ras 2 oncogene in colorectal carcinomas. International Journal of Oncology 3, 841-845.

Kulkarni N \& Reddy BS (1994) Inhibitory effect of Bifidobacterium longum cultures on the azoxymethane-induced aberrant crypt foci formation and fecal bacterial $\beta$-glucuronidase. Proceedings of the Society for Experimental Biology and Medicine 207, 278-283.

Luk GD \& Baylin SB (1984) Ornithine decarboxylase as biological marker in familial colonic polyps. New England Journal of Medicine, 311, 80-83.

Luk GD, Zhang SZ \& Hamilton SR (1989) Effects of timing of administration and dose of difluoromethylomithine on rat colonic carcinogenesis. Journal of the National Cancer Institute 81, 421-427.

McLellan EA, Medline A \& Brid RP (1991) Sequential analyses of the growth and morphological characteristics of aberrant crypt foci: putative preneoplastic lesions. Cancer Research 51, 52705274.

Mukhopadhyay T, Tainsky M, Cavander AC \& Roth JA (1991) Specific inhibition of K-ras expression and tumorigenicity of lung cancer cells by antisense RNA. Cancer Research 51, 17441748.

Parker SL, Tong T, Bolden S \& Wingo PA (1997) Cancer Statistics, 1997. CA-A Cancer J. Clin 47, 5-27.

Pegg AE \& Dolan ME (1989) Investigation of sequence specificity in DNA alkylation and repair using oligodeoxynucleotide substrate. In DNA Repair Mechanisms and their Biological Implications in Mammalian Cells, pp. 45-59. [Lambart MW \& Laval J, editors]. Plenum Press, New York.

Porter CW, Herrera-Ornelas L, Clark J, Pera P. Petrelli NJ \& Mittleman A (1987) Polyamine biosynthetic activity in normal and neoplastic human colorectal tissue. Cancer (Philadelphia) 60, 1275-1281

Pretlow TP, O'Riordan MA, Pretlow TG \& Stellato TA (1992) Aberrant crypts in human colonic mucosa: putative preneoplastic lesions. Journal of Cellular Biochemistry 16G (Suppl.), 55-62.

Reddy BS (1995) Dietary fiber and colon cancer: animal model studies. Preventative Medicine 6, 559-565.

Reddy BS \& Rivenson A (1993) Inhibitory effect of Bifidobacterium longum on colon, mammary and liver carcinogenesis induced by 2 -amino-3-methylimidazo[4,5-f]quinoline, a food mutagen. Cancer Research 53, 3914-3918.

Reddy BS, Engle A, Simi B \& Goldman M (1992) Effect of dietary fiber on colonic bacterial enzymes and bile acids in relation to colon cancer. Gastroenterology 102,1475-1482.

Reddy BS, Hamid R \& Rao CV (1997) Effect of dietary oligofructose and inulin on colonic preneoplastic aberrant crypt foci inhibition. Carcinogenesis 18, 1.371-1374.

Sekine K, Ohta J, Onishi M et al. (1995) Analysis of antitumor properties of effector cells stimulated with a cell wall preparation (WPG) of Bifidobacterium infantis. Biology Pharmaceuticals Bulletin 18,148-153.

Singh J, Kelloff G \& Reddy BS (1992) Effect of chemopreventive agents on intermediate biomarkers during different stages of azoxymethane-induced colon carcinogenesis. Cancer Epidemiology Biomarkers and Prevention 1, 405-411. 
Singh J, Rivenson A, Tomita M, Shimamura S, Ishibashi N \& Reddy BS (1997) Bifidobacterium longum, a lactic acidproducing intestinal microflora inhibit colon cancer and modulate the intermediate biomarkers of colon carcinogenesis. Carcinogenesis, (in press).

Sugimura T, Nagao M, Kawachi T et al. (1977) Mutagen-carcinogens in food, with special reference to highly mutagenic pyrolytic products in broiled foods. In Origins of Human Cancer, pp. 1561-1577. [Hiatt H. Watson JD \& Winsten JA editors]. Cold Spring Harbor. New York.

Sugimura T, Wakabayashi K, Ohgaki H, Takayama S, Nagao MG \& Esumi $H$ (1991) Heterocyclic amines produced in cooked food: unavailable xenobiotics. In Xenobiotics and Cancer, pp. 279-288 [Ernster L, editor]. Japan Science Society Press, Tokyo.

Vivona AA, Shpitz B, Medline A et al. (1993) K-ras mutations in aberrant crypt foci, adenomas and adenocarcinomas during azoxymethane-induced colon carcinogenesis. Carcinogenesis (London) 14, 1777-1781.

Wang X \& Gibson GR (1993) Effect of the in vitro fermentation of oligofructose and inulin by bacteria growing in the human large intestine. Journal of Applied Bacteriology, 75, 373-380.

Wargovich MH, Chen DD, Jimenez A et al. (1996) Aberrant crypts as a biomarker for colon cancer: evaluation of potential chemopreventive agents in the rat. Cancer Epidemiology Biomarkers and Prevention, 5, 355-360. 